abgeschöptt werden. Eine andere Gruppe, zum Beispiel „Oilsink“, sinkt nach der Olaufnahme zu Boden. Diese Stoffe sind abzulehnen. Wenn sie absinken, decken sie den Boden ab und behindern den Stoffaustausch zwischen Boden und Wasser. Auch ist bekannt, daß sie nach einiger Zeit das aufgenommene $O \mathrm{O}$ an das umgebende Wasser wieder abgeben können.

Wal.reäusser: Wird nicht diese Kontaktschicht der Sedimente mit dem abgesunkenen Ol sehr schnell wieder durch neue Sedimente zugedeckt? Wie wirkt es sich dann aus?

Gunket: Ein Überdecken kann normalerweise nur am Strand auftreten. In der freien See ist die Zuwachsrate der Sedimente viel zu gering, um hier irgendeinen. Einfluß zu haben.

MANN: In diesem Zusammenhang sei darauf hingewiesen, daß auch manche Ole am Boden verharzen und dann eine Besiedlung mit Organismen unmöglich machen.

HELLMANN: Ich darf aber dazu noch einmal sagen, daß Absinkmittel immer wieder angeboten werden. Wir haben uns in der Bundesanstalt von Zeit zu Zeit mit ihnen zu befassen. Viele hiervon sind nach geheimgehaltenen Patentrezepten hergestellt worden.

\title{
Ollabbau durch Rein-, Anreicherungs- und Mischkulturen von Bakterien
}

SCHÖBERL: STEWART et al. (1960) haben, soweit mir bekannt ist, lediglich mit einem Bakterienstamm gearbeitet. Sie haben nicht behauptet, daß Paraffine ganz allgemein nur bis zu einem Ester abgebaut würden, sondern sie haben gefunden, daß Alkane mit mehr als $10 \mathrm{C}$-Atomen von dem von ihnen verwendeten Micrococcus-Stamm abgebaut werden. Dabei tritt eine Esteranreicherung in der Kulturlösung auf. Unzählige Arbeiten auf diesem Gebiet haben gezeigt, daß Kohlenwasserstoffe mit weniger als $10 \mathrm{C}$-Atomen bis zu $\mathrm{CO}_{2}$ und $\mathrm{H}_{2} \mathrm{O}$ abgebaut werden.

Schwersfurth: Wie Herr RÜBELT ausführte, haben wir begonnen, uns mit dem Abbau von Kohlenwasserstoffen zu beschäftigen. Für die ersten Versuche mit kohlenwasserstoffabbauenden Mikroorganismen, die den Zweck haben, unseren Chemikern Ausgangsmaterial für organischchemische Gruppenbestimmungen zu liefern und verschiedene Stämme zu isolieren, verwenden wir vorerst Glasröhren, in die unten eine Fritte eingeschmolzen ist. Die Kolben fassen 11 mineralische Nährlösung, die 2 bis $4 \mathrm{ml}$ Kohlenwasserstoff ( $\mathrm{C}_{16}$, Heizöl, Rohöl) erhalten, das auf die Oberfläche gegeben wird. Die Primärkulturen werden mit ölverunreinigtem Boden beimpft. Nach 2 bis 3 Tagen der Belüftung und bei Zimmertemperatur beginnt die Emulgierung der Kohlenwasserstoffe, die jedoch nicht einsetzt, wenn mit Stickstoff begast wird. Die sich entwickelnden Bakterienpopulationen zeigen nicht nur eine Abhängigkeit vom Kohlenwasserstoff, sondern auch vom Alter der Kulturen, woraus gefolgert wird, daß durch den ersten Angriff auf die Kohlenwasserstoffe Stoffwechselprodukte entstehen, die von anderen Bakterienarten in der Folge verwertet werden können. Das Arbeiten mit Mischkulturen hat den Vorteil, daß man hier mit gezielten Isolierungen für spätere Versuche beginnen kann. Limitierende Faktoren jeglichen Abbaues von C-Verbindungen sind - wie wir es auch experimentell im Süßwasser fanden - anorganische N-Quellen und der $\mathrm{PO}_{4}$-Gehalt der Lösungen. Da sich auf die beim Kohlenwasserstoffabbau gebildeten Zwischenprodukte unsere Aufmerksamkeit besonders richten wird, versuchen wir auch, beispielsweise Rohöl, das von der ersten Bakterienpopulation angegriffen wurde, von weiteren Organismengruppen weiter abbauen zu lassen. Mit kontinuierlichen Kulturen kann man hier offensichtlich gut arbeiten. Untersuchungen an Reinkulturen sind zwar später unerläßlich, doch bei dem Versuch, Stoff wechselprodukte chemisch-analytisch zu untersuchen, die nach Olverunreinigungen von Boden und Grundwasser in diesem transportiert werden können, muß Wert auf möglichst konstante Mischpopulationen gelegt werden, um wenigstens einen kleinen Teil der Faktoren des natürlichen Milieus zu imitieren.

SснӧвеRL: Es ist richtig, daß man in der Natur Mischkulturen vorliegen hat. Aber wenn Sie den Versuch unternehmen wollen, Zwischenprodukte des Kohlenwasserstoffabbaus zu identi- 
fizieren, dann müssen Sie mit reinen Stämmen arbeiten, denn Sie müssen wissen, was der einzelne Stamm mit dem vorgelegten Kohlenwasserstoff macht.

GuNkEL: Wenn mich die Umsetzungen im natürlichen Lebensraum interessieren, ich insbesondere wissen möchte, mit welcher Geschwindigkeit eine Verölung durch Abbau "verschwindet", bin ich nicht darauf angewiesen, die Unzahl von Zwischenumsetzungen der" vorhandenen großen Anzahl der Stämme zu untersuchen.

WALlHäUsSER: Lassen Sie mich Herrn SchöвERl unter die Arme greifen! Der Mikrobiologe wird immer danach trachten, auch die Verhältnisse im Reinen zu betrachten. Wir haben, um das gleich zu sagen, sehr große Rückschläge bei Mischkulturen exlebt, weil Sie diese nie ganz in der Hand haben. Diese Mischkulturen gehen heute wunderbar, und dann passiert am nächsten Tag etwas, das Verhältnis zwischen A und B ändert sich, und schon haben Sie ganz andere Bedingungen. Wir haben das bei verschiedenen Mischkulturen beobachtet. Es bleibt uns daher nicht erspart, wenn man hinter dem ganzen Ablauf der Dinge sehen will, doch mit Reinkulturen $\mathrm{zu}$ arbeiten!

GUNKEL: Im natürlichen Lebensraum werden die Umsetzungen - hier also des Olabbaues durch eine große Anzahl von Mikroorganismen, vielleicht 10, vielleicht aber auch 200 oder 500 verschiedene Arten durchgeführt, bzw. ein Teil der Anzahl gehört dieser Lebensgemeinschaft an, ohne Ol abzubauen. Diese Arten stehen miteinander in innigster Wechselbeziehung, sei es als Konkurrenten, sei es, daß sie sich gegenseitig fördern, oder sei es, daß sie sich zeitlich ablösen. Mögen auch einige Arten die „Schrittmacher" sein, so ist es doch ausgeschlossen, von den Reaktionen einer isolierten Art, die jetzt in Reinkultur vorliegt und Umsetzungen im Reagenzglas durchführt, den natürlich vorliegenden Abbau zu erkennen. Es ist notwendig, sowohl Untersuchungen mit Reinkulturen als auch Untersuchungen mit Mischkulturen unter Verhältnissen, die weitmögliche Ahnlichkeit mit denen der Natur haben, durchzuführen. Zugegeben, mit Reinkulturen erhält man eindeutige und stets reproduzierbare Ergebnisse, aber es kommt wohl darauf an, von welchem Blickwinkel her ich das Problem sehe: $\mathrm{Ob}$ ich mich für die Abbaukinetik des Undekans unter konstanten Bedingungen und in der logarithmischen Phase der Vermehrung meines Stammes interessiere, oder ob ich mich für "Über-allesUmsetzungen" eines aus unendlich vielen Verbindungen bestehenden Heizöls interessiere, das eine Verölung des natürlichen Milieus durchgeführt hat. Die ökologische Forschung liegt auf dem Gebiet der Mikrobiologie insbesonders in Deutschland sehr im Argen (vgl. Rheinheimer \& GuNkel 1963). Die wenigen vorhandenen Mikrobiologen arbeiten fast ausschließlich mit Reinkulturen im Labor. Dieses Schwören auf die Reinkultur führte schon zu grotesken Situationen. So wurden für Kläranlagen einer Raffinerie in Ingolstadt Massenkulturen eines Stammes eines ölabbauenden Bakteriums in Holland angezogen und für eine Impfung verwandt. Es trat kein Erfolg ein, trotz Wiederholung. Nadhdem man sich einige Zeit nicht um die Kläranlagen gekümmert hatte, stellte sich die gewünschte Bakterienpopulation von allein ein. Darf ich vielleicht auch hier einmal WAKSMANN (1952) zitieren, er bezieht sich auf die Bodenbakteriologie; dies trifft natürlich entsprechend auch für die Gewässermikrobiologie zu: "General microbiology is based upon the obligatory pure culture method and upon the reactions carried out by these cultures under various conditions. In view of the fact that a specific organism has to compete in a certain process in the soil with numerous other organisms, some of which are much more active and more specialized, the ability of a given organism to carry out a certain function under laboratory conditions and in pure culture is no proof that the organism will carry out the same function in the soil. Stress should be laid on the crude cultures of an elective character, arranged in a manner to allow the observations of the biological activities in the soil itself."

WALLHÄUSSER: Ich stimme vollkommen mit Ihnen überein. Man muß beides nebeneinander herlaufen lassen, beides muß sich ergänzen. Man kann weder auf das eine noch auf das andere verzichten.

Schwersfurth: Bei dem Arbeiten mit Mischkulturen braucht man sich nur über die Tatsache des Vorliegens einer Mischkultur im klaren zu sein. Außderdem ist die Untersuchung der Bedingungen für die Vermehrung in Mischkulturen in der kontinuierlichen Kultur vermutlich sehr interessant, wenn man versucht, durch geringe Milieuänderungen die beiden oder die vielen 
verschiedenen Stämme zu trennen. Außerdem macht das Arbeiten mit Mischkulturen auch manchmal besondere Freude, weil es Uberraschungen geben kann. So finden wir nach etwa 10 Tagen Belüftung in unseren Kolben mit $C_{16}$ so viele Amöben, wie ich sie sonst nur an manchen Kläranlagen an bestimmten Stellen gefunden habe.

SснӧвеRL: Es ist aber trotzdem eine eingestellte Mischkultur, yollkommen gesteuert durch den pH-Wert! Nimmt man eine Reinkultur und gibt so viel Ol auf deren Nährlösung, daß eine Schicht von ca. 0,2 mm entsteht, und schüttelt das Kulturgefäß auf dem Längsschüttler, dann liegt nach etwa drei Tagen eine Emulsion von $\mathrm{Ol}$ in Wasser vor. Das führe ich persönlich auf das Entstehen von Metaboliten, wie zum Beispiel Naphthensäuren zurück, die das Öl emulgieren.

SCHWEISFURTh: Ja, das nehme ich auch an, und LA Rivière (1955a, b) hat das auch schon gewußt. Man könnte an Versuche denken, Olverunreinigungen im Meer durch Zufuhr der limitierenden Verbindungen von $\mathrm{N}$ und $\mathrm{P}$ mikrobiell zu emulgieren und abbauen zu lassen. $\mathrm{Ob}$ in der Praxis der doppelte Effekt der Mikroben schnell genug einsetzt - das ist eine andere Frage.

SснӧвERL: Ja, in diesem Falle muß mit Reinkulturen gearbeitet werden, da wir wissen müssen, welche emulgierenden $Z$ wischenstufen auftreten. Wir können dann vielleicht Emulgatoren herstellen, die eine ähnliche chemische Zusammensetzung wie die der Metaboliten besitzen. Solange wir mit Mischfloren arbeiten, werden wir dieses Ziel nicht erreichen.

Gunkel: Dem stimme ich nicht zu. Ich kann von Bakterien der Mischkultur genauso die gebildeten Emulgatoren bestimmen und untersuchen. Wenn sie von anderen Bakterien so schnell wieder abgebaut werden, daß sie nicht mehr nachweisbar sind, können sie auch keine Wirkung mehr entfalten.

SCHöBERL: Das stimmt nicht unbedingt. Wenn es mir mit einem reinen Stamm gelingt, das Ol durch dessen Abbauprodukte zu emulgieren, so bedeutet das einen großen Schritt vorwärts, was das eigentliche Problem angeht. Dieses Phänomen der "Selbstemulgierung" könnte ich bei Versuchen mit Mischkulturen unter Umständen nicht beobachten, da die betreffenden Verbindungen sofort von anderen Bakterien weiter abgebaut würden.

Gunkel: Können Sie das? Oder ist das jetzt bisher nur ein Wunsch?

SснӧвERL: Ja, ich bin kein Chemiker!

Gunkel: Haben Sie eine Reinkultur und haben Sie das mit OOl gemacht?

SCHÖBERL: Ja!

Gunkex: Mit einem Mischöl, mit einem Heizöl?

SCHÖBERL: Mit einem Rohöl!

WALLHÄUSSER: Betrachten Sie einmal die Zusammensetzung der Bakterienflora im Erdboden, die schwankt während der Jahreszeiten sehr stark, und Sie haben also, wenn Sie jetzt mit einer Mischkultur arbeiten, nicht immer dieselbe Zusammensetzung, sondern Sie haben Mischkulturen in ganz verschiedenen Kombinationen. Diese Verhältnisse müssen Sie berücksichtigen. Ich weiß nicht, inwieweit die Kombinationen im Meer genauso stark variieren wie auf dem Lande. Auf dem Lande können Sie feststellen, daß sich die Zahl der Streptomyceten im Sommer ganz erheblich vergrößert; das Optimum liegt im Juni/Juli, dann ist jedoch ein rascher Rückgang zu beobachten. Das trifft auch für andere Bakterien zu.

GuNKeL: Selbstverständlich verändert sich die Mischkultur in der Natur ständig. Ich habe aber die Aussicht, immer (1) eine Lebensgemeinschaft und (2) stets verschiedene Ollabbauer darin zu haben. Und irgendwie stellen sie sich dann auf die speziellen Bedingungen ein. Verwende ich dagegen eine Reinkultur, gibt es nichts zum Einstellen, weil das Material dazu nicht da ist.

WallhäUsser: Ich wollte nur noch einmal darauf hinweisen, daß seibst, wenn Sie mit Mischkulturen arbeiten, Sie nicht mit ständig gleichbleibenden Verhältnissen rechnen dürfen.

Gunkel: Nein, die Zusammensetzung dieser Mischkulturen schwankt. 
SCHWEISFURTH: Ohne weitere Literaturkenntnisse möchte ich doch meinen, daß es mit Hilfe von kontinuierlichen Kulturverfahren möglich sein sollte, konstante Mischkulturen zu erhalten. Wie schon gesagt, wir haben in Homburg Untersuchungen in dieser Richtung begonnen.

\section{Vermehrung von Mikroorganismen in Oltropfen}

SchweIsfurth: Ich thöchte über erste Versuche zur Frage der Vermehrung von Mikroorganismen in Oltropfen berichten. Bei Versuchen über den mikrobiellen Abbau von Kohlenwasserstoffen (KWS) in von unten belüfteten Kolben fiel als erstes auf, daß nach 2 bis 3 Tagen der Kultur eine Emulgierung stattfindet, bei der anfangs größere Tropfen des jeweils zugefügten Substrates vorlagen, die in der Folge mechanisch, durch vermutlich gebildete oberflächenaktive Stoffe und durch Verzehr weiter verkleinert wurden. Mit zunehmendem Alter der Mischkulturen und der Erschöpfung der N- und $\mathrm{PO}_{4}$-Quellen war der KWS soweit verändert, daß nach Abstellen der Belüftung Rest-Kohlenwasserstoffe teilweise absanken und am Boden liegenblieben.

Da beim Durchströmen der beimptten Flüssigkeitskulturen mit Stickstoff die Bildung von KWS-Tropfen nicht stattfand, eine rein mechanische Zerstörung der auf die Oberfläche der Mineralsalzlösung gegebenen Kohlenwasserstoffe also ausblieb, muß eine Beteiligung von Stoffwechselprodukten der Mikroorganismen vorliegen. Beobachtungen hierzu sind bereits verschiedentlich publiziert worden (LA Rrvière 1955a, b).

Die Tropfenbildung veranlaßte uns zu mikroskopischen Untersuchungen, da es auch von grundsätzlichem Interesse war, festzustellen, ob Mikroben in Oltropfen eindringen können oder ob ein Angriff nur von außen erfolgen kann.

Beim Abbau von $\mathrm{C}_{16}$-Paraffin fiel nach etwa zweitägiger Kulturdauer auf, daß auf den KWSTropfen kleine Tröpfchen lagen. In der gleichen Kultur fanden sich nach 3 bis 4 Tagen massenhaft Oltropfen, deren Oberfläche dicht mit Bakterien besetzt waren. Später traten dann die kugelrunden Oltropfen zurück, es zeigten sich schlierenförmige Gebilde, die nach weiterer Bebrütung zu „Detritus“ zerfielen. In gealterten Flüssigkeitskulturen lagen die Mikroorganismen frei in der Lösung und am Boden vor.

Im Gegensatz hierzu schien nach den ersten Versuchen mit Rohöl ein Eindringen der Bakterien in das OOl relativ schnell zu erfolgen. Ein Beweis für das Eindringen konnte bislang höchstens indirekt geführt werden, da bei der mikroskopischen Untersuchung besiedelten Ols ohne besondere Kunstgriffe nicht schlüssig beurteilt werden konnte, ob die Mikroben nicht doch noch auf der Oberfläche von Olschlieren oder -tropfen lagen.

Bei Kulturen mit Rohöl fiel auf, daß fast alle Bakterien in (oder auf?) den Olschlieren lagen und daß sie auch bei gealterten Kulturen im Bodensatz nur selten frei in der Lösung zu finden waren. Dies könnte für eine Vermehrung der Mikroben im Ol selbst sprechen. Ferner beobachteten wir bei Objektträgerkulturen, daß Öltropfen mit Bakterien innerhalb weniger Sekunden „in“ steriles OOl eindringen und sich die Bakterien in der Folge darin vermehren können.

WallhäUsser: Was sollen die Bakterien ausrichten können, die in den Oltropfen eingedrungen sind?

GUNKEL: HeYER (1966) berichtete über das Wachstum von Bakterien in den wäßrigen Medien. Er ist der Ansicht, daß der Ubertritt in das Ol ein rein physikalischer Vorgang ist, der nicht an die lebende Zelle gebunden ist. Verantwortlich soll ein Uberwiegen hydrophober Gruppen auf der Zelloberfläche sein. Eine Vermehrung soll auch hier fast ausschließlich an der Grenzfläche Öl-Wasser stattfinden, wobei hier die Bakterien die Olseite der Grenzfläche besiedeln.

\section{Isolation von Reinkulturen ölabbauender Bakterien}

THon: Ich möchte über eine Methode berichten, die auf relativ einfache Weise gestattet, Reinkulturen ölabbauender Mikroorganismen zu isolieren. Hiermit kann man Olabbauer auch aus Untersuchungsmaterial gewinnen, in welchem sie in sehr geringer Anzahl vorkommen, ohne 